\title{
Modeling of Human Arm Energy Expenditure for Predicting Energy Optimal Trajectories
}

\author{
L. Zhou ${ }^{1}$ S. Bai ${ }^{1}$ M. R. Hansen ${ }^{2}$ J. Rasmussen ${ }^{1}$ \\ ${ }^{1}$ Department of Mechanical and Manufacturing Engineering, Aalborg University, 9220 Aalborg, Denmark. E-mail: \\ $\{l z h, s h b, j r\} @ m$-tech.aau.dk \\ ${ }^{2}$ Department of Engineering, University of Agder, Grimstad, Norway. E-mail: michael.r.hansen@uia.no
}

\begin{abstract}
Human arm motion can inspire the trajectory planning of anthropomorphic robotic arms to achieve energyefficient movements. An approach for predicting metabolic cost in the planar human arm motion by means of the biomechanical simulation is proposed in this work. Two biomechanical models, including an analytical model and a musculoskeletal model, are developed to implement the proposed approach. The analytical model is developed by modifying a human muscle expenditure model, in which the muscles are grouped as torque providers for computation efficiency. In the musculoskeletal model, the predication of metabolic cost is conducted on the basis of individual muscles. With the proposed approach, metabolic costs for parameterized target-reaching arm motions are calculated and utilized to identify optimal arm trajectories.
\end{abstract}

Keywords: metabolic cost; human arm motion; musculoskeletal model; biomechanics

\section{Introduction}

A human arm has seven dof (degrees-of-freedom) upon basic definition, three in the shoulder, two in the elbow, and two in the wrist. The redundancy in the arm dof implies infinite possible trajectories for a given movement task. For instance, when we pick up a bottle of water, there are a great number of trajectories that the arm can follow. With the hand located at a fixed point, the arm can also have different orientations.

The mechanism behind the selection of the predictable trajectory has been the subject of study over the years. The kinematic analysis (Flash and Hogan, 1985; Atkeson and Hollerbach, 1985) revealed some interesting kinematic features of arm motions, but could not explain the planning mechanism for the activation of the individual muscle. One effective approach to study the planning mechanism is to examine the mechanical and physiological properties of a muscle, and to investigate the behaviour of individual mus- cles in human arm trajectories (Kashima et al., 2002; Fagg et al., 2002; Georgopoulos et al., 1986). Experimental data on multi-joint human arm trajectories obtained from restricted horizontal planar movements have shown that human point-to-point arm motion trajectories have bell-shaped velocity profiles (Abend et al., 1982; Morasso, 1981). Efforts were made to explain the observed trajectories as solutions to optimization problems. Optimization criteria have been proposed including minimum jerk theory (Flash and Hogan, 1985), minimum travel cost theory (Rosenbaum et al., 1995), minimum isometric torque derivative (Kashima and Isurugi, 1998), and averaged specific power (Secco et al., 2005). The criteria used in the optimal trajectory study include also the minimum energy cost hypothesis for human arm trajectories presented and tested by Alexander (1997), among others.

This paper reports our study of human arm in planar motion. Our study focuses on the metabolic energy costs in human arm motions. Two human arm models, 
one analytical and one musculoskeletal, are proposed within our study. In the analytical model, the arm is represented by a 2-dof linkage driven by 4 torque providers (groups of muscles). The muscle metabolism model is modified from a human muscle energy expenditure model proposed by Umberger et al. (2003). The musculoskeletal model is built in the AnyBody ${ }^{T M}$ Modeling System (AnyBody, 2010). Both models are applied to planar arm motion in reaching a group of four targets. For each pair of target points, metabolic energy costs associated to parameterized arm trajectories are simulated and analyzed, from which the optimal arm trajectory for each pair of target points is further identified. The developed models are compared with a model reported in (Alexander, 1997). The analytical model is efficient in calculating energy consumptions and predicting optimal trajectories.

\section{Model of metabolic cost in arm motion}

\subsection{Model of arm}

A simplified arm model is shown in Fig. 1. Confined to planar motion, this arm has only two dof, with the shoulder joint situated at the origin of the coordinate system. The parameters of the arm model are measured or taken from (Winter, 2009), as listed in Table 1. Four target points $P_{1}, P_{2}, P_{3}$ and $P_{4}$ in Fig. 1 are specified, with their coordinates (in meter) being $(0,0.2)$, $(0,0.5),(-0.2,0.3)$ and $(0.2,0.3)$, respectively. Out of these four points, four pairs of starting-end points are established to generate trajectories for arm motions. The combinations of the pairs of points are organized in eight groups, as listed in Table 2, with the arrow indicating the trajectory direction from the starting point to the ending point. Movements between two points from Groups A1 to D1 indicate forward human arm motions, while those from Groups A2 to D2 indicate backward motions.

Table 1: Parameters of the human arm model.

\begin{tabular}{|c|c|c|c|}
\hline Parameter & Value & Unit & Note \\
\hline$m_{1}$ & 2.0 & $\mathrm{~kg}$ & upper arm mass \\
\hline$m_{2}$ & 1.1 & $\mathrm{~kg}$ & lower arm mass \\
\hline$m_{3}$ & 0.4 & $\mathrm{~kg}$ & hand mass \\
\hline$I_{1}$ & 0.021 & $\mathrm{~kg} \cdot \mathrm{m}^{2}$ & upper arm moment of inertia \\
\hline$I_{2}$ & 0.007 & $\mathrm{~kg} \cdot \mathrm{m}^{2}$ & lower arm moment of inertia \\
\hline$l_{1}$ & 0.29 & $m$ & upper arm length \\
\hline$l_{2}$ & 0.23 & $m$ & lower arm length \\
\hline$l_{1 c}$ & 0.14 & $m$ & $\begin{array}{l}\text { distance from centre of mass } \\
\text { to shoulder joint }\end{array}$ \\
\hline$l_{2 c}$ & 0.11 & $m$ & $\begin{array}{l}\text { distance from centre of mass } \\
\text { to shoulder joint }\end{array}$ \\
\hline
\end{tabular}

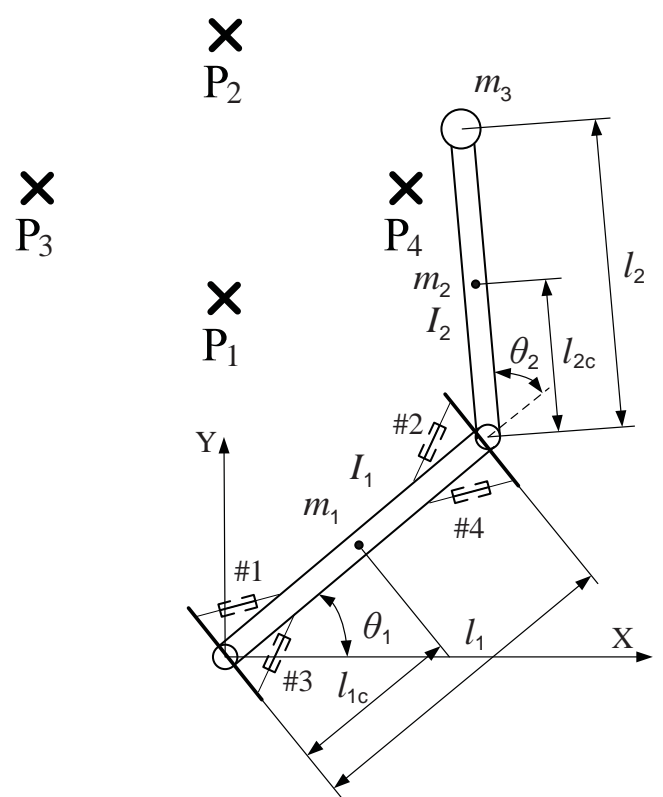

Figure 1: A simplified human arm model.

Table 2: Combinations of the target points.

\begin{tabular}{cccc}
\hline \hline Group A1 & Group B1 & Group C1 & Group D1 \\
\hline$P_{1} \rightarrow P_{2}$ & $P_{3} \rightarrow P_{2}$ & $P_{4} \rightarrow P_{2}$ & $P_{3} \rightarrow P_{4}$ \\
\hline \hline Group A2 & Group B2 & Group C2 & Group D2 \\
\hline$P_{2} \rightarrow P_{1}$ & $P_{2} \rightarrow P_{3}$ & $P_{2} \rightarrow P_{4}$ & $P_{4} \rightarrow P_{3}$ \\
\hline
\end{tabular}

In this arm model, four pieces of torque providers are defined, as shown in Fig. 1. The torque providers, numbered from 1 to 4, stand for the shoulder flexor, the elbow flexor, the shoulder extensor, and the elbow extensor, respectively.

It is assumed that the torque providers inherit the biomechanical properties of individual muscles. Their models can thus be developed by extending the existing muscle models with modifications. The torque providers defined in this study adopt the Hill-type (Hill, 1938) muscle model, with parameters listed in Table 3, where the optimal fiber length $L_{\text {opt }}$ of a torque provider comes from Holzbaur et al. (2005), while the maximum strength-moment $M_{\max }$ exerted on a joint by a torque provider is taken from Chaffin et al. (2006).

\subsection{Model of metabolic costs}

The modeling of muscle metabolic costs is modified from the model of the human muscle energy expenditure proposed by Umberger et al. (2003). Let $\dot{E}$ (in $W a t t / k g)$ be the total energy expenditure rate of a single muscle. It can be expressed as

$$
\dot{E}=\dot{h}_{a}+\dot{h}_{m}+\dot{h}_{s l}+\dot{w}_{c e}
$$


Table 3: Parameters of the torque providers.

\begin{tabular}{ccl}
\hline Torque & $L_{o p t}{ }^{\mathrm{a}}$ & $M_{\max }{ }^{\mathrm{b}}$ \\
Provider No. & {$[\mathrm{cm}]$} & {$[\mathrm{Nm}]$} \\
\hline$\sharp 1$ & 16.2 & 92 \\
$\sharp 2$ & 17.3 & 77 \\
$\sharp 3$ & 27.9 & 67 \\
$\sharp 4$ & 13.4 & 46 \\
\hline a Optimal fiber length from Holzbaur \\
et al. (2005) \\
b Muscle strength moment from \\
Chaffin et al. (2006)
\end{tabular}

where $\dot{h}_{a}$ is the muscle activation heat rate, $\dot{h}_{m}$ is the maintenance heat rate, $\dot{h}_{s l}$ is the shortening/lengthening heat rate and $\dot{w}_{c e}$ is the mechanical power.

In this work, the muscle energy rate $\dot{E}$ is transformed into torque provider power $P$ (in $W a t t)$ as a function of the joint moment and angular velocity, where both the shortening/lengthening heat rate and the mechanical power are included. Assuming that the length of a torque provider can never exceed $L_{\text {opt }}$, the metabolic power of a torque provider becomes

$$
P=-M_{i s o} \omega+G_{s} \frac{\rho}{\sigma} \frac{M_{\max }}{L_{o p t}} \omega
$$

where the first term stands for the mechanical power and the second one stands for the heat rate due to shortening/lengthening. In Eq. (2), $M_{i s o}$ is the moment exerted on a joint by the muscle isometric force, and $\omega$ is the joint angular velocity. Moreover, $\sigma$ is a specific tension, which takes a value of $0.25 \mathrm{MPa}$, as recommended by Umberger et al. The muscle density for mammalian muscle is $\rho=1059.7 \mathrm{~kg} \cdot \mathrm{m}^{-3}$ (Mendez and Keys, 1960).

The coefficient $G_{s}$ (in $N / k g$ ) of Eq. (2) is a factor of the muscle shortening/lengthening heat rate (Umberger et al., 2003), which depends on the percentage of fast twitch fibres (FT), and the shortening or lengthening of the muscle. When a muscle is shortening, the factor is calculated by

$$
G_{s}=0.0323 \times \% F T-8.33
$$

As an example, when a muscle with $50 \%$ fast twitch fibres is shortening, $G_{s}=-6.72 \mathrm{~N} / \mathrm{kg}$; and when the muscle is lengthening, it takes the value $G_{s}=$ $33.3 \mathrm{~N} / \mathrm{kg}$.

The isometric moment $M_{\text {iso }}$ is the moment exerted by the muscle. This moment can be obtained by modifying the mechanical joint moment with respect to the angular velocities of joints (Leeuwen, 1991). The iso- metric moment $M_{\text {iso }}$ can be calculated as

$$
M_{i s o}= \begin{cases}\frac{M\left(1+K r_{\omega}\right)}{1-r_{\omega}} & \text { for shortening } \\ \frac{M\left(1-7.56 K r_{\omega}\right)}{1-0.8 r_{\omega}-13.6 K r_{\omega}} & \text { for lengthening }\end{cases}
$$

where $r_{\omega}=\omega / \omega_{\max }$ with $\omega_{\max }$ being the angular velocity corresponding to the maximum shortening speed of a muscle. $\omega_{\max }$ is set as $22 \mathrm{rad} / \mathrm{s}$ for flexor, and $28 \mathrm{rad} / \mathrm{s}$ for extensor, according to Winters and Stark (1985). The constant $K$ depends on the muscle fibre type. Consequently, the metabolic power of a muscle becomes a function of joint angular velocity $\omega$ and joint moment $M$.

The joint mechanical moment can be calculated for shoulder and elbow joints, separately. In planar motion, the moment at the shoulder joint, $M_{s}$, is calculated as

$$
\begin{aligned}
M_{s}= & x_{1} m_{1} \ddot{y}_{1}+x_{2} m_{2} \ddot{y}_{2}+x_{3} m_{3} \ddot{y}_{3} \\
& -y_{1} m_{1} \ddot{x}_{1}-y_{2} m_{2} \ddot{x}_{2}-y_{3} m_{3} \ddot{x}_{3} \\
& +I_{1} \dot{\omega}_{1}+I_{2}\left(\dot{\omega}_{1}+\dot{\omega}_{2}\right)
\end{aligned}
$$

where $\left(x_{1}, y_{1}\right),\left(x_{2}, y_{2}\right)$, and $\left(x_{3}, y_{3}\right)$ specify the centres of mass of the upper arm, the lower arm, and the hand, respectively. The angular accelerations of the shoulder joint and the elbow joint are $\dot{\omega}_{1}$ and $\dot{\omega}_{2}$, respectively.

Likewise, the moment at the elbow joint, $M_{e}$, is given as

$$
\begin{aligned}
M_{e}= & \left(x_{2}-x_{e}\right) m_{2} \ddot{y}_{2}+\left(x_{3}-x_{e}\right) m_{3} \ddot{y}_{3} \\
& -\left(y_{2}-y_{e}\right) m_{2} \ddot{x}_{2}-\left(y_{3}-y_{e}\right) m_{3} \ddot{x}_{3} \\
& +I_{2}\left(\dot{\omega}_{1}+\dot{\omega}_{2}\right)
\end{aligned}
$$

where $\left(x_{e}, y_{e}\right)$ are the coordinates of the elbow joint.

\subsection{Parameterized arm motion}

To describe all the possible arm motions, a Fourier series of joint angular velocities is considered

$\omega=a_{1} \sin \left(\frac{\pi t}{T}\right)+a_{2} \sin \left(\frac{2 \pi t}{T}\right)+a_{3} \sin \left(\frac{3 \pi t}{T}\right)+\cdots$

where $a_{1}, a_{2}$, and $a_{3}$ are coefficients, $T$ is the duration of the motion. Since the angular velocity has to be zero at $t=0$ and $t=T$, only sine terms are included in this Fourier series form.

In our study, we use the first two sine terms of Fourier series to approximate angular velocity. Assuming $\theta_{0}$ and $\theta_{T}$ be the angles of a certain joint at the $t=0$ and $t=T$, the angular velocity can be expressed with two-term Fourier series as

$$
\omega=\frac{\pi}{T}\left[\frac{\theta_{T}-\theta_{0}}{2} \sin \left(\frac{\pi t}{T}\right)+D \sin \left(\frac{2 \pi t}{T}\right)\right]
$$


where $D$ is the deviation of a certain joint angle, following Alexander's definition (Alexander, 1997). Hereby, $D_{s}$ denotes the shoulder angle deviation, and $D_{e}$ the elbow angle deviation.

By changing the angle deviation factor $D$ for both shoulder and elbow joints, different trajectories between the same pair of target points can be generated. Integrating both sides of Eq. (8), leads to

$\theta=\frac{\theta_{T}-\theta_{0}}{2}\left[1-\cos \left(\frac{\pi t}{T}\right)\right]+\frac{D}{2}\left[1-\cos \left(\frac{2 \pi t}{T}\right)\right]+\theta_{0}$

The angle of the shoulder $\theta_{1}$ can vary from $-45^{\circ}$ to $150^{\circ}$, and the angle of the elbow $\theta_{2}$ can vary from $0^{\circ}$ to $150^{\circ}$.

The joint angular acceleration is obtained by differentiating Eq. (8)

$$
\dot{\omega}=\left(\frac{\pi}{T}\right)^{2}\left[\frac{\theta_{T}-\theta_{0}}{2} \cos \left(\frac{\pi t}{T}\right)+2 D \cos \left(\frac{2 \pi t}{T}\right)\right]
$$

\section{Musculoskeletal model}

The torque providers used in the analytical model of the arm motion in Sec. 2 represent groups of muscles. It is desirable to extend the study to individual muscles and investigate the behavior of the muscles at the musculoskeletal level. To this end, we developed another model by taking advantage of a state-of-the-art biomechanical modeling system, namely, the AnyBody Modeling System (AnyBody, 2010).

A musculoskeletal right arm model was built in the AnyBody ${ }^{\mathrm{TM}}$ Modeling System, as shown in Fig. 2. The whole musculoskeletal model is comprised of 39 joints and 134 muscles. The model is derived from the repository models in AnyBody and each muscle unit is modeled using a three element Hill-type muscle model. In this study, as the model arm is confined to planar motion, only glenohumeral flexion joint and elbow flexion joint of the arm are free to move, and the others are constrained. In addition to the AnyBody model, a MatLab program was developed to control the changes of the parameters and trajectories.

For human analytical musculoskeletal models, Hilltype muscle models are almost exclusively used. Hilltype muscle models consist of a contractile element (CE) that generates force and represents the muscle fibers, and a passive element (PE) in parallel with $\mathrm{CE}$, and the above two modeling in series with a serial elastic element $(\mathrm{T})$, as depicted in Fig. 3.

In the AnyBody modeling system, the mechanical power of an individual muscle-tendon unit is calculated by

$$
P_{\text {mech }}=f_{T} v_{M T}=f_{T} i_{M T}
$$

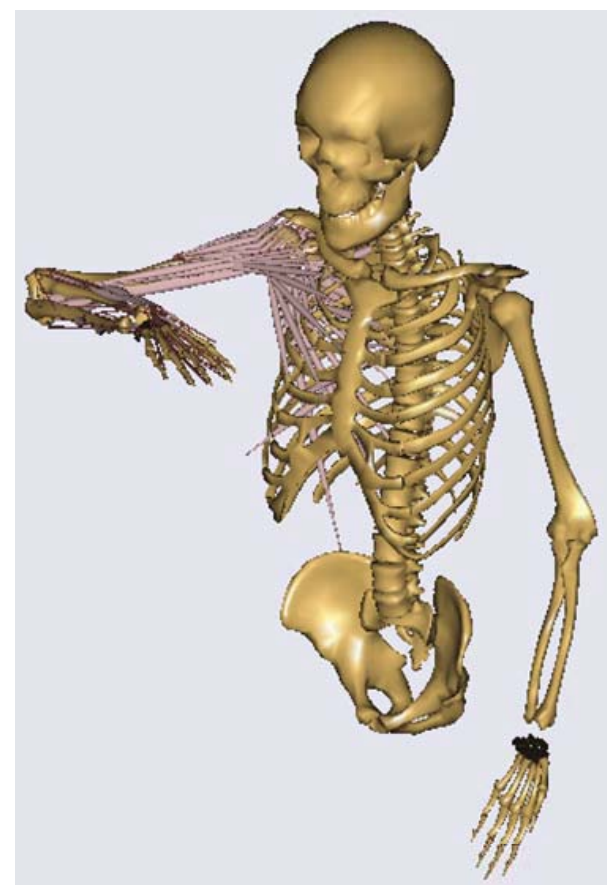

Figure 2: A human musculoskeletal model built with the AnyBody Modeling System.

The prediction of metabolic power is based on the efficiency of the contractile element in AnyBody as

$$
P_{m}=\frac{P_{\text {mech }}}{\mu}, \quad \begin{cases}\mu=0.25 & \text { for shortening } \\ \mu=-1.2 & \text { for lengthening }\end{cases}
$$

\section{The Alexander's model (reference model)}

We compared our models with a model reported by Alexander (1997), which is introduced briefly. The method of calculating metabolic power in Alexander's model made use of the work of Ma and Zahalak (1991). The metabolic power of a uniarticular muscle is given by

$$
P_{\text {ref }}=M_{i s o} \omega_{\max } \Phi\left(r_{\omega}\right)
$$

where $M_{i s o}, \omega_{\max }$ and $r_{\omega}$ follow the definitions in Eq. (4). The data fit function $\Phi$ was adopted from Ma and Zahalak's work, with the form as

$$
\Phi\left(r_{\omega}\right)= \begin{cases}0.23-0.16 \exp \left(-8 r_{\omega}\right) & \omega \geq 0 \\ 0.01-0.11 r_{\omega}+0.06 \exp \left(23 r_{\omega}\right) & \omega<0\end{cases}
$$

The Alexander's model consists of two antagonistic pairs of muscles to drive the arm. At any time, only one muscle of each pair is active. The Alexander's model uses mechanical power scaled by a function $\Phi$ as the 


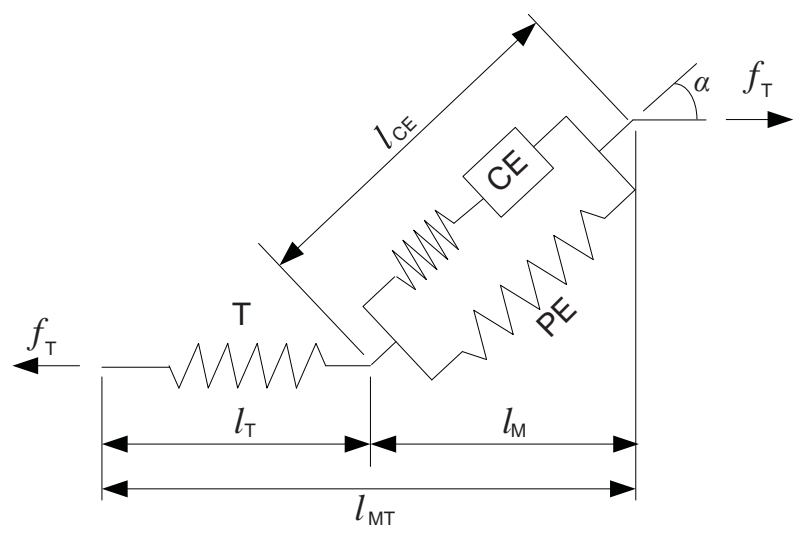

Figure 3: Hill-type muscle model consisting of three elements with parallel-series arrangement.

main source of metabolic power for an individual muscle. While only two muscles are considered to be active, the reference model underestimates the metabolic power of human arm motion. In contrary, the analytical model we propose includes mechanical power and muscle shortening/lengthening heat together as the metabolic power. The analytical model utilizes torque providers working as muscle groups instead of only four muscles in the reference model. Our anatomical musculoskeletal model consists of 134 muscles, which is an extremely detailed model of the human arm.

\section{Simulation routine}

When running simulation with the analytical arm model, only one torque provider of each joint would be activated. The metabolic power of each torque provider is calculated using Eqs. (2)-(10). The metabolic energy of an individual torque provider is obtained by integrating the metabolic power over the entire duration. The metabolic costs of all individual torque providers are added together to obtain the total metabolic energy cost.

In the musculoskeletal arm model, all the muscles associated with arm motion are active. In each simulation, the program will write a file containing the joint angles. The program in Matlab runs the AnyBody console application in batch mode to conduct inverse dynamic analysis on the musculoskeletal arm. The metabolic powers of all the muscles are summed to obtain the overall metabolic power of the whole arm model. The metabolic cost can be obtained by integrating the overall metabolic power over the motion duration. The simulation routine of coupling Matlab with AnyBody is shown in Fig. 4.

For the analytical model, both the shoulder angle de-

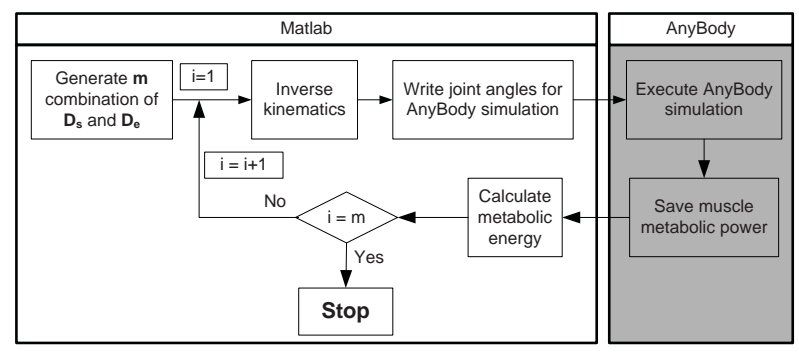

Figure 4: Simulation routine of the musculoskeletal model in the AnyBody software.

viation $D_{s}$ and the elbow angle deviation $D_{e}$ are varied from $-40^{\circ}$ to $40^{\circ}$ in step size of $1^{\circ}$. For the musculoskeletal model, $D_{s}$ and $D_{e}$ are varied from $-40^{\circ}$ to $40^{\circ}$ in step size of $5^{\circ}$.

\section{Simulation results}

Biomechanical simulations are conducted on the two proposed models and the reference model as well. Simulation results of metabolic power, metabolic energy consumption, and optimal trajectories are compared to each other among the three models.

\subsection{Predicted metabolic power}

The metabolic powers predicted by the analytical, musculoskeletal and reference models are shown in Fig. 5. Four trajectories are selected from Groups A1 to D1, respectively. All trajectories are specified by $D_{e}=$ $-20^{\circ}$ and $D_{s}=20^{\circ}$.

There is barely experimental data about metabolic costs in human arm motion, due to lack of experimental techniques. We cannot compare the simulation results to the experiments to validate the model in a holistic level. However, there is much literature about experimental results and models on a single muscle (Umberger et al., 2003; Ma and Zahalak, 1991). Since we can simulate the actions of all the muscles in a musculoskeletal arm, the whole metabolic cost for an arm can be calculated as well. The musculoskeletal model is a much detailed human arm model with 134 muscles, which means that the musculoskeletal model predicts the metabolic power consumptions as the sum of the metabolic power of each individual muscle.

In general, the analytical model predicts metabolic power similar to the musculoskeletal model. There is a good agreement between the analytical and musculoskeletal models in the rates of change, referring to the increasing and declining parts of the metabolic power curves in Fig. 5. The reference model predicts similar metabolic power profile to the analytical model, but 


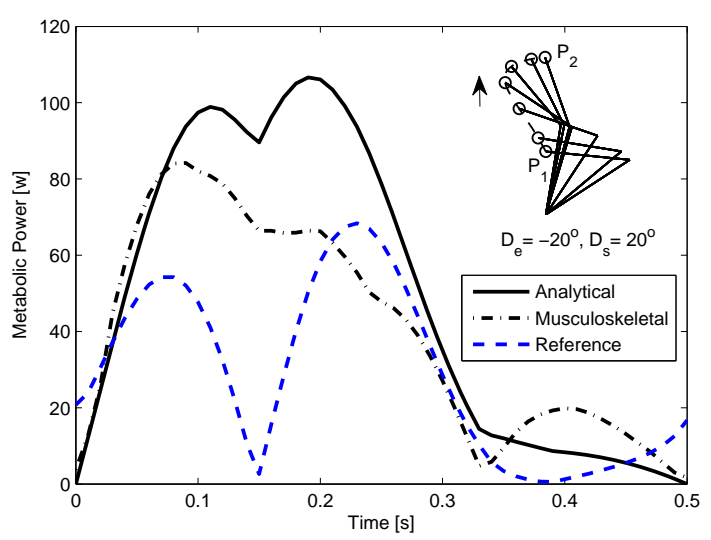

(a)

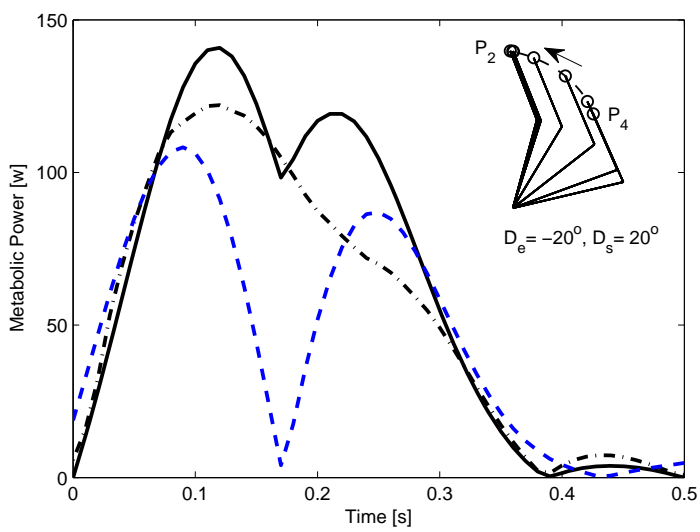

(c)

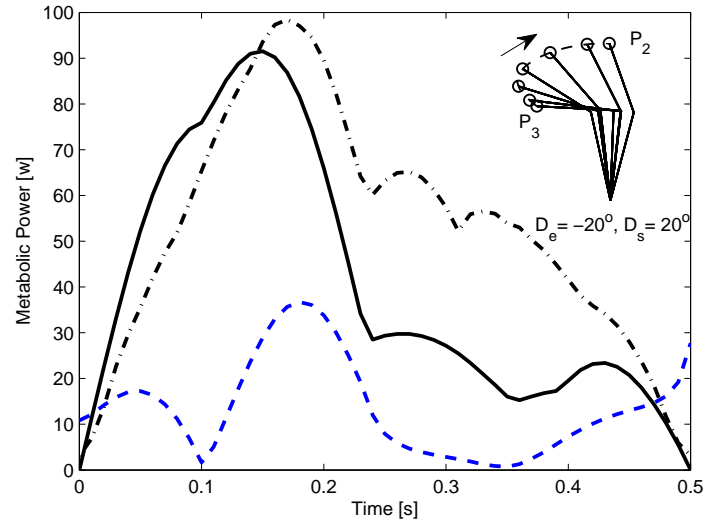

(b)

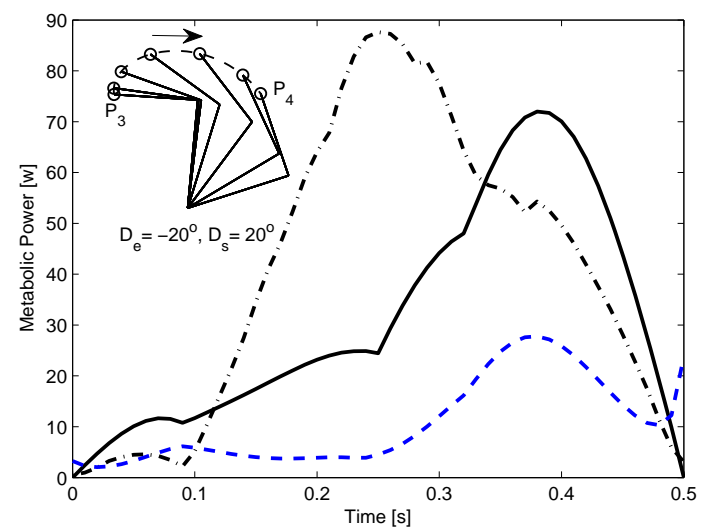

(d)

Figure 5: Metabolic powers of arm motion with four trajectories.

the maximum values are far below the two proposed models. The analytical model is more close to the musculoskeletal model in predicting metabolic power compared to the reference model.

\subsection{Predicted metabolic energy}

The metabolic energy cost of the analytical model for the parameterized trajectories in Group A1 is shown in Fig. 6(a), while those of the musculoskeletal model and the reference model (Alexander, 1997) are shown in Fig. 6(b) and 6(c), respectively.

The duration of movements is $0.5 \mathrm{~s}$. In Fig. 6, each point $\left(D_{e}, D_{s}\right)$ represents a trajectory. The global minimum is marked by a red cross. For the analytical model moving in Group A1, the minimum energy cost is found at $D_{e}=-7^{\circ}$ and $D_{s}=0^{\circ}$, with a minimal value of $17.662 \mathrm{~J}$. For the musculoskeletal model moving in Group A1, the minimum is found at $D_{e}=0^{\circ}$ and $D_{s}=10^{\circ}$, with a minimal metabolic energy cost of 14.764J. The CPU time for calculating minimal energy cost for the three models is list in Table 4. It is seen that the analytical model developed in this work is more efficient compared with the other two models.

Table 4: Computational time for the three models.

\begin{tabular}{ll}
\hline Model & Elapsed time $[\mathrm{s}]$ \\
\hline Analytical & 20 \\
Musculoskeletal & $7.13 \times 10^{4}$ \\
Reference & 37 \\
\hline
\end{tabular}

\subsection{Optimal trajectories}

Based on the metabolic energy cost contour, the optimal trajectories can be identified. The optimal trajectories predicted by the proposed two models and the reference model are shown in Fig. 7 for comparison. The optimal trajectories obtained with different 


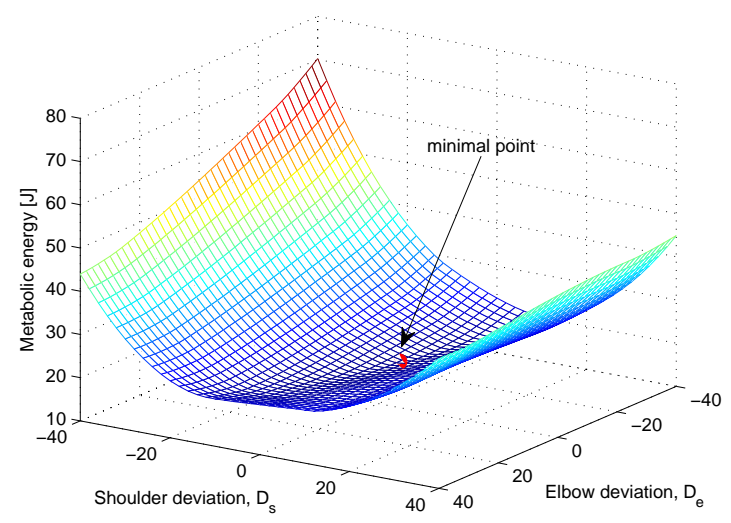

(a)

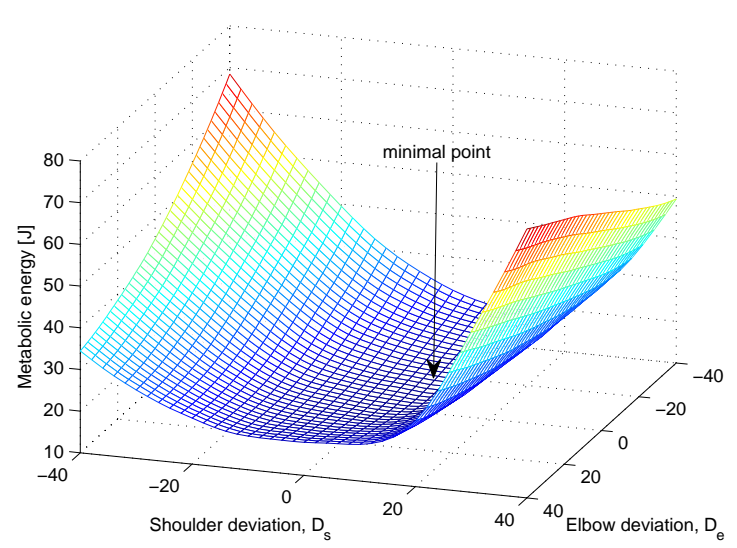

(b)

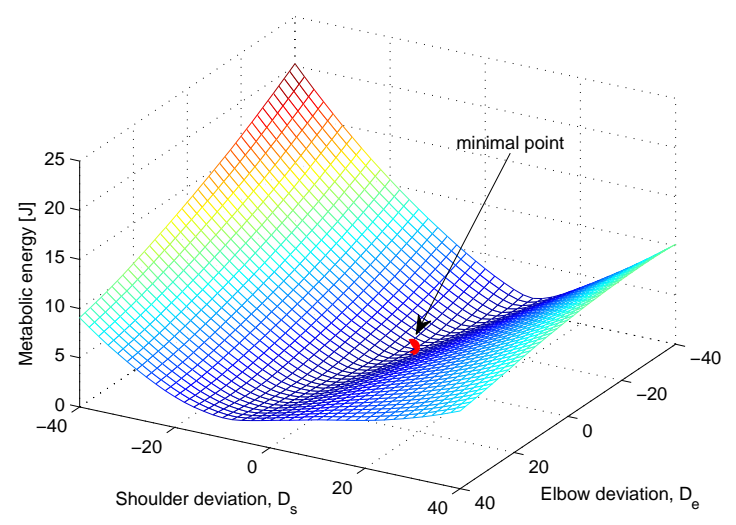

(c)

Figure 6: Metabolic energy cost contours obtained with different models, (a) the analytical model, (b) the musculoskeletal model, (c) the reference model reported in (Alexander, 1997).
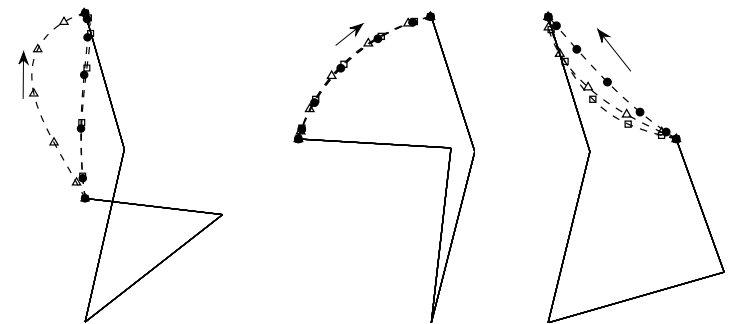

(a) Group A1

(b) Group B1

(c) Group C1
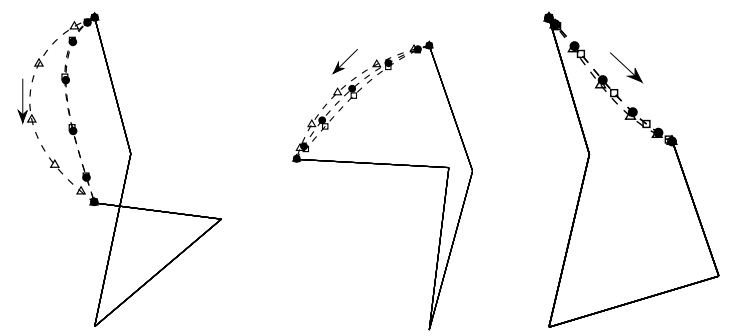

(d) Group A2

(e) Group B2

(f) Group C2

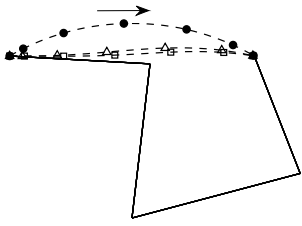

(g) Group D1

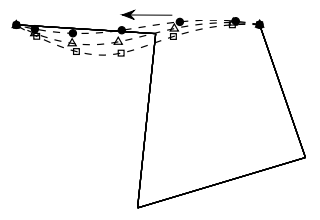

(h) Group D2
Figure 7: Optimal trajectories predicted by the proposed two models and a reference model (Alexander, 1997). The hand paths are marked with dots $(\bullet)$, triangle $(\triangle)$, and box symbols for the proposed analytical, musculoskeletal and reference models, respectively.

models are marked with specified symbols. Optimal trajectories for both forward and backward arm motions are displayed. The dashed line indicates the path of the hand, and the moving direction is marked by the arrow. The elbow $\left(D_{e}\right)$ and shoulder $\left(D_{s}\right)$ angle deviations and metabolic energy costs $\left(E_{m}\right)$ for optimal trajectories in Fig. 7 are summarized in Table 5. The elbow and shoulder joint velocities for the optimal trajectories from Groups A1 to D1 are depicted in Fig. 8, while Fig. 9 shows the corresponding hand velocities.

The two proposed models predict very similar optimal trajectories with respect to the reference model, even though the reference model greatly underestimates the metabolic energy consumption. There are some discrepancies of the optimal trajectories 


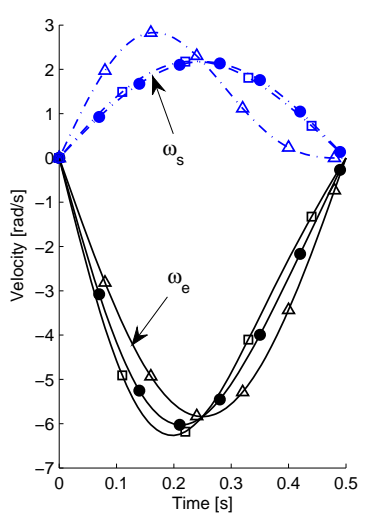

(a) Group A1

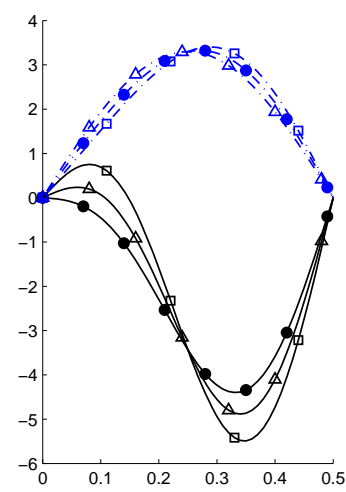

(c) Group C1

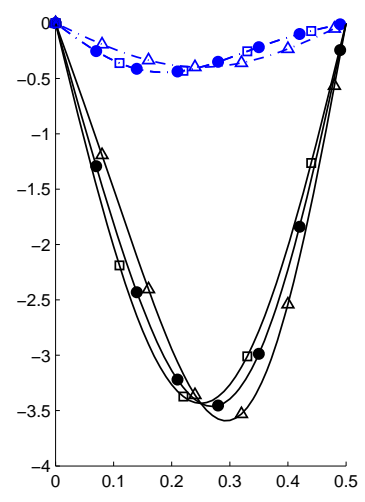

(b) Group B1

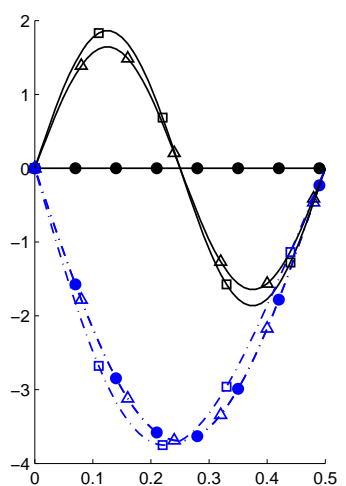

(d) Group D1

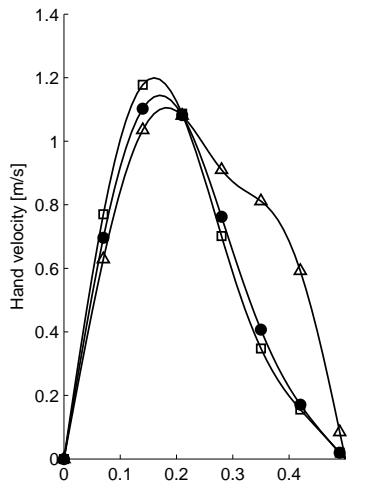

(a) Group A1

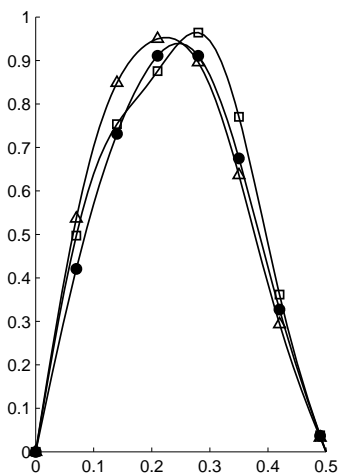

(c) Group C1

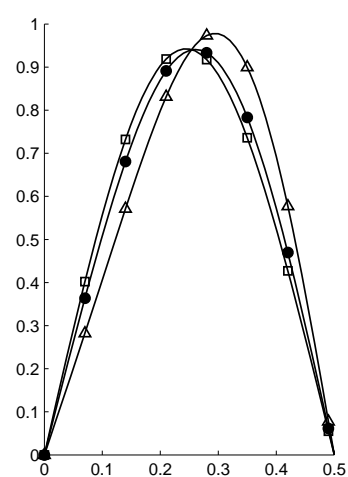

(b) Group B1

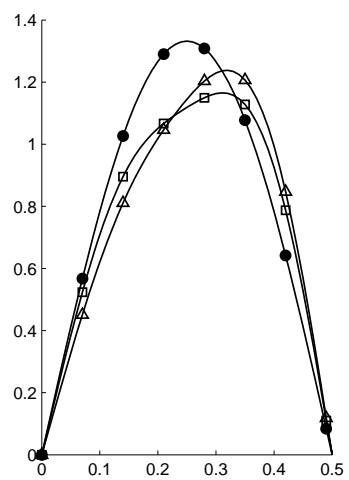

(d) Group D1

Figure 8: Angular velocities of elbow joint $\left(\omega_{e}\right.$, solid curve) and shoulder joint ( $\omega_{s}$, dashed curve) corresponding to optimal trajectories for motions of Groups A1, B1, C1 and D1 in Fig. 7. The velocity curves are marked with $\operatorname{dots}(\bullet)$, triangle $(\triangle)$, and box symbols for the proposed analytical, musculoskeletal and reference models, respectively.

predicted by the musculoskeletal model and the other two models in Groups A1 and A2. This may be due to the deviations of the musculoskeletal model.

The hand velocities of the optimal trajectories show bell-shape profiles, which have a good agreement with the straight arm motion observed by Flash and Hogan (1985). Fig. 7 shows that unconstrained point-to-point motions are not only approximately straight, but also metabolic energy optimal.

The optimal trajectories in forward and backward arm movements, displayed in Fig. 7(a)-7(c) and Fig. 7(d)-7(f), respectively, show that they do not necessarily follow the same hand paths in forward and backward movements.

Figure 9: Hand velocities of the corresponding optimal trajectories of Groups A1, B1, C1 and D1 in Fig. 7. The velocity curves are marked with dots $(\bullet)$, triangle $(\triangle)$, and box symbols for the proposed analytical, musculoskeletal and reference models, respectively.

\subsection{Metabolic power of optimal trajectories}

Metabolic power of the optimal trajectories for target points in Groups A1 and C1 are calculated by the two proposed models and the reference model, as shown in Fig. 10. The metabolic power is calculated based on the optimal trajectories obtained by the analytical model. It is seen that the analytical and the musculoskeletal model predict similar profile of metabolic power consumption, even though the analytical model overestimates the power a bit than the musculoskeletal one. Nevertheless, the model reported by Alexander underestimates the metabolic power significantly compared to the two proposed models. 
Table 5: Elbow $\left(D_{e}\right)$ and shoulder angle deviation $\left(D_{s}\right)$ and metabolic cost $\left(E_{m}\right)$ of optimal trajectories in Fig.7.

\begin{tabular}{|c|c|c|c|c|c|c|c|c|c|c|c|c|}
\hline \multirow{2}{*}{ Model } & \multicolumn{3}{|c|}{ Group A1 } & \multicolumn{3}{|c|}{ Group B1 } & \multicolumn{3}{|c|}{ Group C1 } & \multicolumn{3}{|c|}{ Group D1 } \\
\hline & $D_{e}$ & $D_{s}$ & $E_{m}$ & $D_{e}$ & $D_{s}$ & $E_{m}$ & $D_{e}$ & $D_{s}$ & $E_{m}$ & $D_{e}$ & $D_{s}$ & $E_{m}$ \\
\hline Analytical & $-7^{\circ}$ & $0^{\circ}$ & $17.662 \mathrm{~J}$ & $2^{\circ}$ & $-1^{\circ}$ & $6.816 \mathrm{~J}$ & $15^{\circ}$ & $-2^{\circ}$ & $22.934 \mathrm{~J}$ & $0^{\circ}$ & $0^{\circ}$ & $6.939 \mathrm{~J}$ \\
\hline Musculoskeletal & $0^{\circ}$ & $10^{\circ}$ & $14.764 \mathrm{~J}$ & $5^{\circ}$ & $0^{\circ}$ & $3.537 \mathrm{~J}$ & $20^{\circ}$ & $0^{\circ}$ & $24.594 \mathrm{~J}$ & $15^{\circ}$ & $0^{\circ}$ & $14.505 \mathrm{~J}$ \\
\hline \multirow[t]{2}{*}{ Ref. Model } & $-11^{\circ}$ & $1^{\circ}$ & $2.614 \mathrm{~J}$ & $0^{\circ}$ & $-1^{\circ}$ & $1.46 \mathrm{~J}$ & $26^{\circ}$ & $-4^{\circ}$ & $10.929 \mathrm{~J}$ & $17^{\circ}$ & $-3^{\circ}$ & $1.422 \mathrm{~J}$ \\
\hline & \multicolumn{3}{|c|}{ Group A2 } & \multicolumn{3}{|c|}{ Group B2 } & \multicolumn{3}{|c|}{ Group C2 } & \multicolumn{3}{|c|}{ Group D2 } \\
\hline Analytical & $-1^{\circ}$ & $5^{\circ}$ & $28.792 \mathrm{~J}$ & $5^{\circ}$ & $-3^{\circ}$ & $20.980 \mathrm{~J}$ & $17^{\circ}$ & $-4^{\circ}$ & $19.982 \mathrm{~J}$ & $20^{\circ}$ & $0^{\circ}$ & $23.751 \mathrm{~J}$ \\
\hline Musculoskeletal & $15^{\circ}$ & $10^{\circ}$ & $14.429 \mathrm{~J}$ & $5^{\circ}$ & $0^{\circ}$ & $5.875 \mathrm{~J}$ & $20^{\circ}$ & $-5^{\circ}$ & $14.670 \mathrm{~J}$ & $25^{\circ}$ & $0^{\circ}$ & $26.340 \mathrm{~J}$ \\
\hline Ref. Model & $-3^{\circ}$ & $6^{\circ}$ & $3.916 \mathrm{~J}$ & $10^{\circ}$ & $-5^{\circ}$ & $4.862 \mathrm{~J}$ & $17^{\circ}$ & $-4^{\circ}$ & $2.045 \mathrm{~J}$ & $30^{\circ}$ & $-2^{\circ}$ & $12.113 \mathrm{~J}$ \\
\hline
\end{tabular}

\subsection{Different durations with the analytical model}

Optimal trajectories in Group A1 for four different durations $(0.3 \mathrm{~s}, 0.5 \mathrm{~s}, 0.8 \mathrm{~s}, 1 \mathrm{~s})$ predicted by the analytical model are shown in Fig. 11. Details of the optimal trajectories with the four time durations are listed in Table 6.

Table 6: Elbow and shoulder angle deviations of optimal trajectories with different durations predicted by the analytical model.

\begin{tabular}{lcccccccc}
\hline \multirow{2}{*}{ Points } & \multicolumn{2}{c}{$0.3 \mathrm{~s}$} & \multicolumn{2}{c}{$0.5 \mathrm{~s}$} & \multicolumn{2}{c}{$0.8 \mathrm{~s}$} & \multicolumn{2}{c}{$1 \mathrm{~s}$} \\
\cline { 2 - 8 } & $D_{e}$ & $D_{s}$ & $D_{e}$ & $D_{s}$ & $D_{e}$ & $D_{s}$ & $D_{e}$ & $D_{s}$ \\
\hline Group A1 & $-7^{\circ}$ & $0^{\circ}$ & $-7^{\circ}$ & $0^{\circ}$ & $-3^{\circ}$ & $-1^{\circ}$ & $24^{\circ}$ & $-12^{\circ}$ \\
Group B1 & $2^{\circ}$ & $-1^{\circ}$ & $2^{\circ}$ & $-1^{\circ}$ & $2^{\circ}$ & $-1^{\circ}$ & $2^{\circ}$ & $-1^{\circ}$ \\
Group C1 & $16^{\circ}$ & $-3^{\circ}$ & $15^{\circ}$ & $-2^{\circ}$ & $15^{\circ}$ & $-2^{\circ}$ & $15^{\circ}$ & $-2^{\circ}$ \\
Group D1 & $0^{\circ}$ & $0^{\circ}$ & $0^{\circ}$ & $0^{\circ}$ & $0^{\circ}$ & $0^{\circ}$ & $0^{\circ}$ & $0^{\circ}$ \\
\hline
\end{tabular}

For motions in Groups B1, C1 and D1, all the optimal trajectories for four different durations are almost identical, according to the values of $D_{e}$ and $D_{s}$ in Table 6 .

In Group A1, the optimal trajectories of the fast movements $(0.3 \mathrm{~s}, 0.5 \mathrm{~s})$ and those of the slow movements $(0.8 \mathrm{~s}, 1 \mathrm{~s})$ show a nearly straight path of the hand, as that Flash and Hogan (1985) observed. No significant differences are observed.

\section{Discussions}

It can be noticed that all models, the analytical and musculoskeletal models and the reference model, lead to very similar optimal trajectories. Also, the modelpredicated optimal trajectories match the observed human planar arm movements (Flash and Hogan, 1985).

The analytical model takes a simple form with only four torque providers included, compared to the musculoskeletal model using 134 muscles. On the other hand, the metabolic energy costs calculated by the two proposed models and reference model have considerable differences, as shown in Table 5. In some cases, the muscles in a human arm are antagonistic to causing substantial energy waste. This part of energy cost is considered in the proposed analytical model and the musculoskeletal model, but not in the reference model. It is apparent that the metabolic costs calculated by the two models developed presently are much larger than that calculated by the reference model. In some cases, the metabolic cost calculated by the analytical model is even a bit larger than that by the musculoskeletal model. It is reasonable that the musculoskeletal model is considered as a better predictor of metabolic energy cost.

Predicted optimal trajectories in all groups are similar among the three models, except Groups A1 and A2. The differences are apparent if we look into the elbow angle deviation $D_{e}$ and shoulder angle deviation $D_{s}$ in Table 5. The elbow angle deviation $D_{e}$ differences among Groups B1, C1, D1 is below $17^{\circ}$, and the shoulder angle deviation $D_{s}$ differences is below $4^{\circ}$. Comparing forward and backward arm motions like Groups B1 and B2, the optimal trajectories of the forward motions (Group B1) are similar to those of the backward motions (Group B2). However, the optimal trajectories of forward motions in Group A1 and backward motions in Group A2 are different, especially those predicted by the analytical and reference models. The trajectories predicted by the two models in Fig. 7(a) show almost straight paths, and those in Fig. 7(d) show apparent curved paths.

Besides, the computational times with the analytical and musculoskeletal models are significantly different. The analytical model involves only numerical calculation, and is much more efficient than the musculoskeletal model. A natural progression of the work is to investigate whether a joint moment-driven model, which can be even more computationally efficient, can also provide valid results, thus eliminating the need for muscles in the model. 


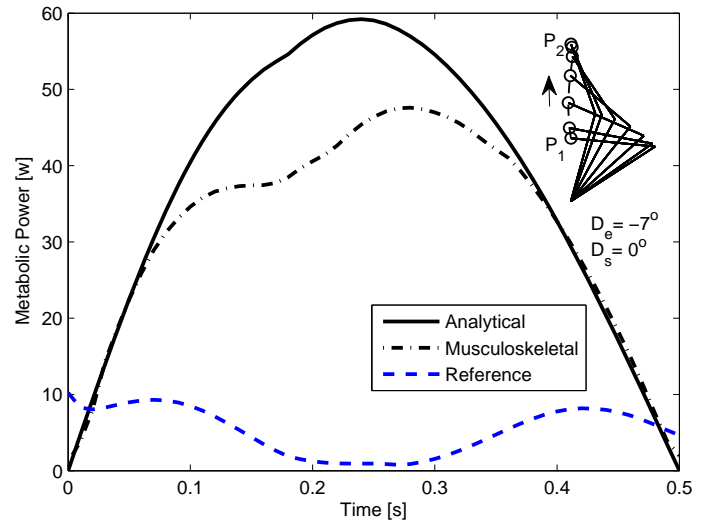

(a) Group A1

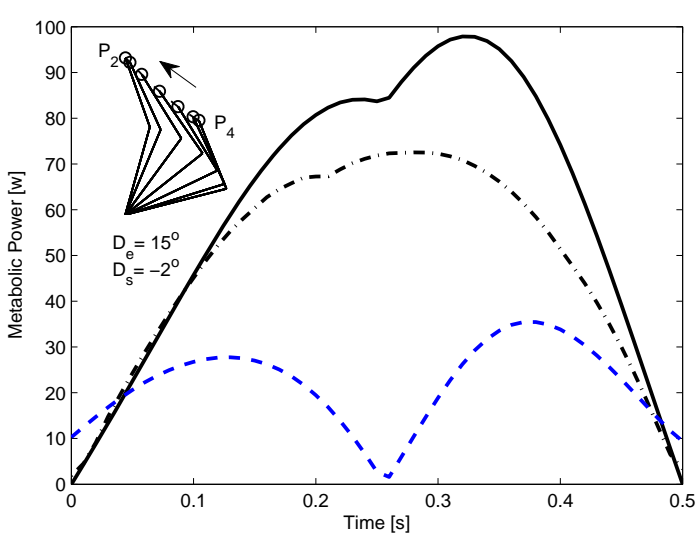

(b) Group C1

Figure 10: Metabolic power of optimal trajectories in Groups A1 and C1 predicted by the analytical, musculoskeletal and reference models.

\section{Conclusions}

In this paper, an analytical and a musculoskeletal models were developed and compared with a reference model. Both models are able to calculate metabolic cost. Optimal trajectories were identified with the developed models for planar arm movements. The comparisons between the three models showed that even though the two proposed models and the reference model predict similar optimal trajectories, the reference model greatly underestimates the metabolic cost. Among the two newly proposed models, the musculoskeletal model is more accurate in predicting the metabolic energy cost while the analytical one is more efficient in predicting optimal trajectories. Given the fact that the predication results from two models are very close, the simple analytical model can replace the complicated musculoskeletal model in predicting

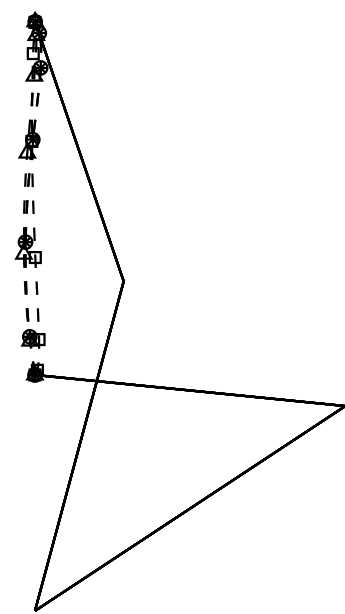

Figure 11: Optimal trajectories in Group A1 corresponding to four different durations by the proposed analytical model. The hand paths are marked with dots $(0)$, cross $(\times)$, triangle $(\triangle)$, and box symbols for duration 0.3 $\mathrm{s}, 0.5 \mathrm{~s}, 0.8 \mathrm{~s}$, and $1 \mathrm{~s}$, respectively.

metabolic costs and energy efficient trajectories in certain applications where the overall behavior of the arm motion is of interest.

\section{References}

Abend, W., Bizzi, E., and Morasso, P. Human arm trajectory formation. Brain, 1982. 105:331-348. doi:10.1093/brain/105.2.331.

Alexander, R. M. A minimum energy cost hypothesis for human arm trajectories. Biological Cybernetics, 1997. 76(2):97-105. doi:10.1007/s004220050324.

AnyBody. AnyBody Modeling System. 2010. Available from: www. anybodytech.com.

Atkeson, C. G. and Hollerbach, J. M. Kinematic features of unrestrained vertical arm movements. Journal of Neuroscience, 1985. 5(9):2318-2330.

Chaffin, D. B., Andersson, G. B. J., and Martin, B. J. Occupational Biomechanics. John Wiley \& Sons, New York, 4 edition, 2006.

Fagg, A. H., Shah, A., and Barto, A. G. A computational model of muscle recruitment for wrist movements. Journal of Neurophysiology, 2002. 88:33483358. doi:10.1152/jn.00621.2002. 
Flash, T. and Hogan, N. The coordination of arm movements: An experimentally confirmed mathematical model. Journal of Neuroscience, 1985. $5(7): 1688-1703$.

Georgopoulos, A. P., Schwartz, A. B., and Kettner, R. E. Neuronal population coding of movement direction. Science, 1986. 233:1416-1419. doi:10.1126/science.3749885.

Hill, A. V. The heat of shortening and the dynamic constants of muscle. Proceedings of the Royal Society of London, Series B, Biological Sciences, 1938. 126(843):136-195. doi:10.1098/rspb.1938.0050.

Holzbaur, K. R. S., Murray, W. M., and Delp, S. L. A model of the upper extremity for simulating musculoskeletal surgery and analyzing neuromuscular control. Annals of Biomedical Engineering, 2005. 33(6):829-840. doi:10.1007/s10439-005-3320-7.

Kashima, T. and Isurugi, Y. Trajectory formation based on physiological characteristics of skeletal muscles. Biological Cybernetics, 1998. 78:413-422. doi:10.1007/s004220050445.

Kashima, T., Isurugi, Y., and Shima, M. An optimal control model of a neuromuscular system in human arm movements and its contrl characteristics. Artificial Life Robotics, 2002. 6:205-209. doi:10.1007/BF02481269.

Leeuwen, J. L. V. Optimum power output and structural design of sarcomeres. Journal of Theoretical Biology, 1991. (149):229-256. doi:10.1016/S00225193(05)80279-6.

Ma, S. and Zahalak, G. I. A distribution-moment model of energetics in skeletal muscle. Journal of
Biomechanics, 1991. 24(1):21-35. doi:10.1016/00219290(91)90323-F.

Mendez, J. and Keys, A. Density and composition of mammalian muscle. Metabolism, Clinical and Experimental, 1960. (9):184-188.

Morasso, P. Spatial control of arm movements. Experimental Brain Research, 1981. 42:223-227. doi:10.1007/BF00236911.

Rosenbaum, D. A., Loukopoulos, L. D., Meulenbroek, R. G. J., Vaughan, J., and Engelbrecht, S. E. Planning reaches by evaluating stored postures. Psychological Review, 1995. 102(1):28-67. doi:10.1037/0033-295X.102.1.28.

Secco, E. L., Valandro, L., Caimmi, R., Magenes, G., and Salvato, B. Optimization of two-joint arm movements: a model technique or a result of natural selection? Biological Cybernetics, 2005. 93:288-306. doi:10.1007/s00422-005-0003-2.

Umberger, B. R., Gerritsen, K. G. M., and Martin, P. E. A model of human muscle energy expenditure. Computer Methods in Biomechanics and Biomedical Engineering, 2003. 6(2):99-111. doi:10.1080/102558403100009167.

Winter, D. A. Biomechanics and motor control of human movement. John Wiley \& Sons, New Jersey, 4 edition, 2009.

Winters, J. M. and Stark, L. Analysis of fundamental human movement patterns through the use of indepth antagonistic muscle models. IEEE Transactions on Biomedical Engineering, 1985. 32:826-839. doi:10.1109/TBME.1985.325498. 